\title{
A Hybrid Algorithm using Grey Wolf and Chicken Swarm for Flexible Job Shop Scheduling
}

\author{
S.Kavitha, P.Venkumar
}

\begin{abstract}
The Job Shop Scheduling Problem (JSSP) is one of the major inconvenience models because it is the hardest combinatorial change in nature. So far number of algorithms developed to solve JSSP and still there is scope to develop an efficient algorithm. Hence, this paper considered a unique hybrid algorithm which is the combination of the Grey Wolf and Chicken Swarm Optimization algorithm with the objective of minimization of makespan time and cost. The most extraordinary compilation (makespan), and the tardiness is considered in the meantime. The hybrid algorithm count relies upon the reenactment of the swarming behavior of wolves' individuals. The base objectives of each wolf and swarms from most bewildering thicknesses of the group are considered as the objective work for the improvement. The results show that the proposed hybrid Grey Wolf and Chicken Swarm Optimization algorithm can obtain better results in terms of various iteration levels. The accuracy and robustness are also applied in real industrial conditions and for large size problems. The proposed method results show that very less time duration when compared with the Genetic Algorithm (GA) and Ants Colony Optimization (ACO) algorithm.
\end{abstract}

Keywords : Job Shop Scheduling Problems, Grey Wolf Optimization, Chicken Swarm Optimization, Hybrid Algorithm

\section{INTRODUCTION}

In industrial equipment's, most of the complex problems are arriving, in that Job Shop Scheduling (JSSP) is very much important in most of the cases. To solve such problems various research was done during the past recent days. In that, the basic JSSP is that some machines do the same process on several jobs. As per Gonçalves et al. (2005), JSSP is cleared that it is the process of scheduling ' $N$ ' number of operations on ' $M$ ' machines [1]. Each job subsists of numerous tasks that must be handled in an order to achieve the minimum makespan. A new suggestion must be demonstrated with the prediction of response for an adequate and fundamental condition to attain its feasibility. In this way, an infeasible arrangement will be disposed of instantly and the enhanced basic way calculation is connected to play out the neighborhood scan for an achievable arrangement. With this motivation, various flexible job shop scheduling problem (FJSSP) methods were started in the mid-1990s.

Revised Manuscript Received on December 05, 2019.

* Correspondence Author

First Author Name, S.Kavitha, Department of Mechanical Engineerig, School of Automotive and Mechanical Engineering ,Kalasalingam Academy of Research and Education Krsihnankovil, Tamilnadu, India, kavitha@klu.ac.in

Second Author Name*, P.Venkumar, Department of Mechanical Engineerig, School of Automotive and Mechanical Engineering, Kalasalingam Academy of Research and Education, Krsihnankovil, Tamilnadu, India, venkumar@klu.ac.in
The emerging FJSSP grows even the traditional algorithms like a branch and obligated algorithms were provided an acceptable solution for job scheduling problems [2]. Although FJSSPs is one of the most important in the real-time because most of the research suggested that FJSSP is in the infancy stage. The suggested concept is to utilize the resource without wasting unnecessary computational time. In machinery, the process of each task is important because it decides the critical factor in product wise outcome. To get an exact outcome, the resources are to be properly applied in a short time. It is based on the algorithmic concepts and its classifications. This research reviewed various algorithms and standards with the above concepts. Never expect optimal solutions from approximate modules and it is defined with the help of minimizing the concept of adapting the new solutions and techniques in a given time. The following heuristic algorithms [3] and meta-heuristic algorithms [4] are the two different classes of algorithms considered for enriching the knowledge on job shop scheduling.

Many research efforts have concentrated on the development of efficient methods for FJSSP. However, since the FJSSP is strangely difficult to hard problems in polynomial time, only medium-size problems can be solved within a reasonable time by the exact algorithm. The approximate and heuristic methods make a trade-off between solution quality and computational cost. In most of the cases, makespan is one of the terms determined to enrich the JSSP because it is the highest finishing point of each ' $N$ ' job. It is considered to indicate the purpose of the JSSP as a good schedule by considering all the ' $\mathrm{M}$ ' machines [5].

Recently, most of the research literature has focused on a swarm-based algorithm to get into the solutions for JSSP. The concept of the discrete solution spaces in scheduling problems clarified with the particle swarm position representation and particle movement. But existing techniques are suffering from the problem of immature convergence and easily trapped into a local optimum. Hence, this research focuses on the combination of the Grey Wolf (GWO) and Chicken Swarm Optimization (CSO) algorithm to extract the solution by attaining the parallel strategy. Further, this research is discussed with the literature survey in next section II. The identified problems are identified with the recent methodology concepts under section III. Then, the algorithmic outcomes are illustrated in section IV. Finally, the research is summarized in section $\mathrm{V}$. 


\section{LITERATURE REVIEW}

The separation or identification of job shop scheduling is one of the difficult processes in traditional algorithms. In such cases, a GA is applied by Gonzalez et al. (2008) for JSSP. It is determined with the fitness value directly depending upon the setup time of the jobs [6]. In such cases, there is a need for the setup that mus keep some time for assigning some specific duration with each machine and apply before another job enters into the same machine. To avoid all timing problems and merge fitness value is mixed by the Hybrid GA and local search were considered.

Ponsich and Coello (2013) [7] described a hybrid differential evolution-Tabu search algorithm (DE-TS) is the solution for the Job Search Scheduling problem. In the predefined process, the algorithm is effective and its establishment is difficult to predict the real-time challenges. Hence, there is a need for considering hybrid algorithms and predict the effective computational rate. In such an aspect, these DE-TS are deployed to solve the JSSP. Similarly, some other hybrid genetic search strategies are considered in job shop scheduling processes like hybrid genetic and variable neighborhood descent algorithm and a hybrid genetic algorithm and bottleneck shifting, Tabu Search (TS) and Simulated Annealing (SA). The computational tests in regard to 100 or more JSSP occurrences outlined that their novel cross breed DE-TS calculation was better in connection than other cutting edge techniques. The results likewise uncovered that the certainty temporary width was lesser than $0.7 \%$ of the reference makespan yield esteem, confirming the low dispersion of the arrangements was acquired.

Kassu and Eshetie (2015) utilized a heuristic calculation called moving bottleneck, which diminishes occupations from the bottleneck machines [8]. It prevailing to improve add up to makespan. Then again, Cheng et al. (2016) built up a calculation in light of water weeds proliferation rule to settle JSP [9]. The conduct of how the water weeds look for water sources is received to construct a swarm-insight like calculation. Heuristic calculation ended up having aggressive viability and productivity contrasted with a traditional another calculation in JSSP. Zhou and Feng (2001) proposed a hybrid experience heuristics Genetic algorithm is the solution for Job Search Scheduling problem, proposed concept considers the following major factors such as Shortest Processing Time (SPT), number of Jobs, Remaining Work (MWKR) [10]. Park et al., (2003) described to solve job scheduling problems using the hybrid Genetic algorithm, in which the works schedules have effectively handled a priority-based set of rules and the hybrid genetic algorithm (HGA) is priority job and then the experience-based local search heuristic algorithm improves the solution [11].

Sakawa and Kubota (2000) targeted to solve fuzzy logic-based time and date [12]. The ablest regions of a selected area strengthen the search space for GA is frequently inaccessible and along these lines, an effective GA calculation should combine with another meta-heuristic calculation. Lin et al., (2010) presented a PSO algorithm to solve and compared with SA algorithm [13]. It consists of a multi-objective type and individual enhancement method for solving, flexible job shop scheduling problems, the result of PSO more efficient and robust compared with SA. Zhang et al., (2013) targeted their efforts at significantly scaling down the total weighted tardiness in JSSP [14]. In perspective of the extraordinary difficulty, the counterfeit honey bee state $(\mathrm{ABC})$ calculation was gotten for settling the issue. An area nature of the issue was recognized, and from that point, innovative calculation was intended to enhance the use abilities of the ABC. According to the exhaustive computational results, their inventive procedure was observed to be exceptionally compelling in settling the activity shop planning issue with the general weighted benchmarks delay.

Sha and Lin (2010) proposed, a multi-objective PSO, to increase the search quality and efficiency of searching for optimal scheduling time and jobs [15]. Muthiah et al., (2016) [16] proposed the JSSP became flexible when using the Hybridization of ABC and PSO Algorithm for to effectively reduce time and cost for multi-assigned jobs. Motivated by this concept, we propose a novel intelligent algorithm for the cost problems based on the Hybridization of ABC and PSO. Particle swarm optimization is an evolving method based on swarm intelligence. This method can be classified as an iterative and stochastic type. Wisittipanich and Kachitvichyanukul (2012) proposed a concept makespan problem to solve using two enhanced differential evolution algorithms for, minimize job scheduling makespan tardiness by doing an equal balance of analysis and consumption ability in differential evolution algorithms [17]. Ziaee et al., (2014) proposed Job shop scheduling with makespan objective: A heuristic approach, this method minimizing the makespan of jobs for JSSP and discovered a solution for good schedules frequently [18].

Ma et al., (2014) targeted Hybrid Particle Swarm Optimization and Simulated Annealing (SA) Algorithm for JSSP, developed by a hybrid algorithm for improving the makespan of jobs of searching by using PSO and SA has been used for local search optimization solution [19]. Asadzadeh et al., (2015) proposed a local search genetic algorithm investigates the various benchmark instances and their performance of the proposed approach and thought to be an individual from vast unmanageable numerical issues called the NP-hard [20]. The hereditary calculations were successfully utilized in a large group of booking issues, particularly in the activity shop planning. The hybridization spoke to a productive system of improving the execution and proficiency of the hereditary calculations. The benchmark occasions were used to assess the execution of the recommended strategy. The cheering results showed the way that their imaginative operator based nearby inquiry hereditary calculation could fundamentally upgrade the effectiveness. The objective is to enhance the current circumstance by moving parts, as per predefined rules, to accomplish the popular general arrangement.

Liu et al (2015) splendidly propelled the estimation of dissemination calculation which concentrated on viably handling the post-changing test [21]. An upgraded EDA was planned by conveying a large group of components disregarded in the first EDA, 
which enveloped the recorded ideal arrangement and the homogeneous arrangement vectors. The improved calculation was named as the quick estimation of circulation calculation (FEDA) as it could show a model execution in the merging velocity and calculation precision, of the first EDA. To put it plainly, the brilliant transformation and the proficient FEDA calculation offered a successful and practical approach to viably handle the dynamic and adaptable fluffy employment shop booking issue.

Chang et al. (2015) proposed concept provides correct solutions for the makespan problem using Hybrid Taguchi-genetic Algorithm (HTGA) correct jobs at the correct time [22]. Marichelvam et al (2018) presented the HLOA compare with two benchmark data sets, and the results to be improved in the FJSSP. It consists of single objective and enhanced the method to solve in flexible job shop scheduling problems. [24] And also he presented HGSSA consists of multiobjective considering the makespan and the flowtime in this chapter[25].

However, some methods are still defected and thus much makespan work, such as multi-objective, FJSSP can solve effective proposed HTGA. Above the literature study about job scheduling according to the complete computational results, their inventive procedure was expected to observe to be exceedingly viable in settling the activity shop booking issue with the aggregate weighted different benchmarks and different occupations.

\section{Methodology}

In this research, a hybrid job shop scheduling problemsolving algorithm is considered with the objective of minimizing makespan. The job shop scheduling problem under study can be enlightened as follows; given number of ' $n$ ' jobs $J=\left[J_{1}, J_{2}, \ldots ., J_{n}\right]$ each of which consists of numeral of ' $h$ ' operations $\mathrm{O}=\left\{\mathrm{O}_{1}, \mathrm{O}_{2}, \ldots, \mathrm{O}_{\mathrm{h}}\right\}$ that should be execute on various machines with a specific end goal to be finished. The activities of a given task should be performed on an arrangement of ' $\mathrm{m}$ ' foreordained machines $\mathrm{M}=\{\mathrm{M} 1, \mathrm{M} 2 \ldots$ $\mathrm{Mm}$ \} while satisfying arrangements. In this issue, every task should be prepared on ' $m$ ' machines, ' $\mathrm{h}$ ' activities with the end goal that its handling time is need-based. The undertaking considered here is to discover the stage of the procedure on the hardware to lessen the planning span or makespan with a specific end goal to expand machine usage in different occupations and examinations the different calculations adequately use various machines.

The Job Shop Scheduling Problem (JSSP) is an appendix of the common problem which empowers an assignment to be handled by machines in a pre-organized set. The critical capacity is to apportion every single undertaking to a machine and to sort out the elements of the machines, all together that the most extreme length of finish (makespan) of the whole errand is decreased to the base. The JSSP can be characterized as takes after given various jobs (n) and the number of machines for an activity, every ' $n$ ' work has been prepared on ' $m$ ' machines with no interferences. Every task must be done on m machines for a settled span. Each machine can process at the most extreme of one activity at any given moment. On the off chance that activity once started on a solitary machine for its preparing, at that point it must finish its handling before taking its second procedure. Two activities are impossible at a solitary time, however, a similar need level is given for each activity on each task. There is no sit out of gear time and no separation time on every activity. Every activity is to be handled on various machines, in a given request. To identify the various problems in a prescribed job reduces the according to time to complete that job. The proposed method using the Hybridization of the GWO and CSO techniques is utilized to minimize the makespan times within the optimal scheduling. The GWO and CSO algorithms are swam intelligent based optimization algorithms.

\section{A. Grey Wolf Algorithm}

Grey wolf (Canislupus) derived from the Canidae family. The grey wolves are best carnivore occupied the major element in the environmental food chain. In an avergage 5-12 wolves are considered as a group. For algorithmic representations, a very strict social dominant denoted by $\alpha, \beta$, $\delta$, and $\omega$ pecking order is represented as shown in Fig.1. It represents the effects of a $2 \mathrm{D}$ place vector and some of the possible neighbors are adorned in Fig. 2. From the figure, grey wolf and attacking a target in the place first position denoted $(\mathrm{X}, \mathrm{Y})$ can the updated position between target and the grey wolves denoted of $\left(\mathrm{X}^{*}, \mathrm{Y}^{*}\right)$. The various places around the suitable agent of the grey wolf can be attacked concerning the current target other agents wait for an attack by adjusting the vector value. For the updated position of the instance, denoted by $\left(\mathrm{X}^{*}-\mathrm{X}, \mathrm{Y}^{*}\right)$ can be reached by setting and the possible renew positions of a grey wolf in 3D space.

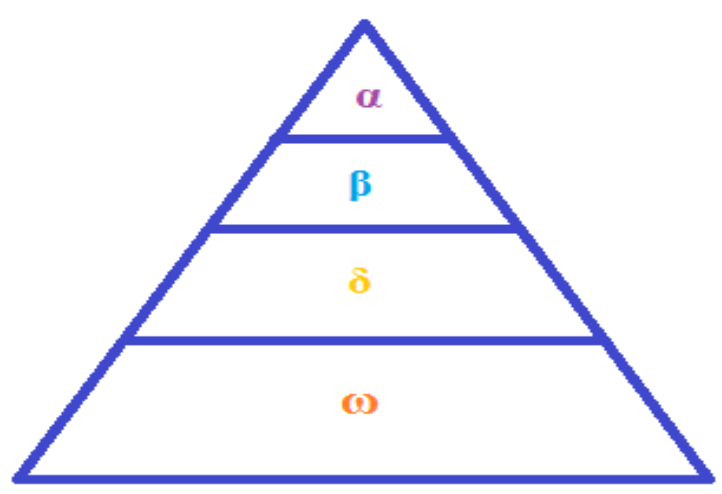

Fig. 1. Grey wolf top down approach

The hunting process is usually calculated by alpha $(\alpha)$ might also take part in hunting every current position $(\beta)$ and an updated position $(\delta)$. However, a proposed system the objective is to consider the search space with the best attack target. In order to exactly obtain the hunting activity of grey wolves, best candidate solution- $\alpha$, current position- $\beta$, and then new position- $\delta$ have better ability about the possible place of hunting. 


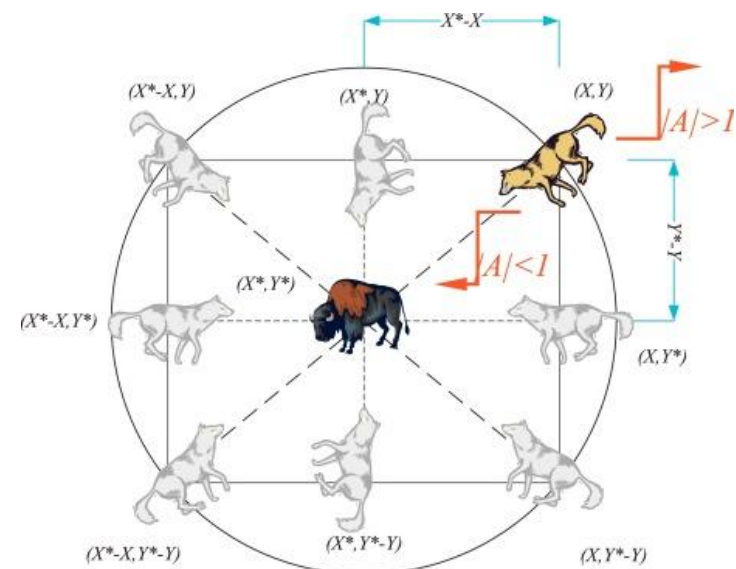

Fig. 2. Hunting position between grey wolves and target

$$
\begin{aligned}
& \vec{D}=\left|\vec{C} \cdot \vec{x}_{p}(t)-\vec{x}(t)\right| \\
& \vec{X}(t+1)=\vec{x}_{P}(t)-\vec{A} \cdot \vec{D} \\
& \vec{A}=2 \vec{a} \cdot \overrightarrow{r_{1}}-\vec{a} \\
& \vec{C}=2 \cdot \overrightarrow{r_{2}} \\
& \vec{x}(t+1)=\frac{\vec{x}_{1}+\vec{x}_{2}+\overrightarrow{x_{3}}}{3}
\end{aligned}
$$

The GWO algorithm decides to search agents assumed wolves to renew their position based on the place of the variables Alpha, Delta, Gama and Beta attack towards the target omega hunting prey. Within encircling proposed mechanisms need to reduce number operators.

\section{B. Chicken Swarm Optimization Algorithm}

Chicken swarm optimization is initially proposed by Mengby Shanghai University in 2014 [23]. It is a kind of examining optimization algorithm with better concurrence efficiency and robustness. It is a sort of looking at streamlining calculation with better simultaneousness effectiveness and strength. The idea selected behind this CSO is that chicken group is partitioned into numerous gatherings as indicated by their rank request and rummaging capacity to ensure each gathering comprises of a rooster and a few hens and chickens, and the aggressive connection between different gatherings and different levels of chickens. As per the natural attributes of chicken, the conduct of every chicken from the part of the scientific computation is controlled. By and large, the chicken has the more grounded and most elevated capacity to discover nourishment, requested by the hen, at last, chicks have the most noticeably awful searching capacity to sustenance gathering limit. In the case, virtual chickens $(\mathrm{N})$ are presented with the chicken population. It is considered with the time $(\mathrm{t})$ and its positions represented with the mathematical equations

$$
x_{i j}^{t}(i \varepsilon[1, \ldots N], j \varepsilon[1, \ldots N])
$$

From equation (6), the spatial dimension (D) of a chicken is determined with the food gathering process.

The movement behaviors of cock mathematical function representation as

$$
\begin{aligned}
& x_{i, j}=x_{i, j}\left(1+\operatorname{Randn}\left(0, \sigma^{2}\right.\right. \\
& \sigma^{2}=\left\{\begin{array}{c}
1, f_{i} \leq f_{k} \\
\frac{f_{k}-f_{i}}{e\left|f_{i}\right|+\varepsilon}, \text { else }
\end{array} k \in[1, N], k \neq i\right.
\end{aligned}
$$

Rand $\left(0, \sigma^{2}\right)$ is the Gaussian distribution function means a function that finds value and the standard deviation is $\sigma^{2} . \varepsilon$ is used to avoid the zero division error which is the smallest constant in a calculation. $k$ Is an index of a rooster by randomly choose from a group of cocks and $f$ is the corresponding fitness value of rooster.

Hens followed by the cock and gather its food. Additionally, the hens will be hidden by chickens, they can also arbitrarily steal food by other chickens. Their behaviors of hens are represented mathematically represented in the following equation (9).

$$
\begin{aligned}
& x_{i, j}^{(t+1)}=x_{i, j}^{t}+s_{1}^{*} \operatorname{Rand}\left(x^{t} r_{1_{j}^{j}}-x_{i, j}^{t}\right)+s_{2^{*}} \operatorname{Rand}\left(x_{r_{2, j}}^{t}-x_{i, j}^{t}\right) \\
& s_{1}=e^{f_{i}-f_{r_{1}}} \\
& s_{2}=e^{f_{r_{2}}-f_{i}}
\end{aligned}
$$

From the equation, Rand is a standardized random number in $[0,1] . r_{1} \varepsilon[1 \ldots . . . N]$ it's rooster's index, and $r_{2} \varepsilon[1$, ..., $\mathrm{N}]$ hen's group mates index is the calculated index value of the chicken, which is randomly chicken certain from their group and represent the relation of randomly selected chicken $\mathrm{r}_{1} \neq \mathrm{r}_{2}$.

Finally, the movement behavior of the chicks is formulated as the equation (12).

$$
x_{i, j}^{(t+1)}=x_{i, j}+F L *\left(x_{m, j}^{t}-x_{t, j}^{t}\right)
$$

$x^{t m}, j(m \varepsilon[1, \ldots n])$ signify the position of the Number of 'i' chick's mother and $F L(F L \varepsilon[0,2])$ is a parameter, that defined the hen will collect the food and chicks follow their mother. Chick's food searching strategy considering individual differences (FL) of each chick will follow the mother to gather its food randomly selected between 0 and 2 .

The Job shop scheduling time taken of CSO is better than other PSO, and also its concurrence process is divided into 3 stages. Initially, the cock search rules are considered for 
search feasible solution and then using the hens and chicks to optimize and reduce the time to improve the accuracy. The details of the hybrid algorithm is shown in Table I.

Table I-: Hybrid Algorithm Details

\begin{tabular}{|l|l|l|l|}
\hline \multicolumn{4}{|c|}{ Problem statement 1} \\
\hline Method & $\begin{array}{l}\text { BEST } \\
\text { COST }\end{array}$ & $\begin{array}{l}\text { TIME } \\
\text { (sec) }\end{array}$ & BEST SOLUTION \\
\hline Genetic Algorithm & 154 & 28.72 & $3,6,5,2,4,4,5,1,4,2,1,6,2,5,1,3$ \\
\hline $\begin{array}{l}\text { Ant Colony } \\
\text { Optimization }\end{array}$ & 168 & 26.06 & $6,5,2,4,3,1,6,3,6,2,2,1,3,4,4,5$ \\
\hline $\begin{array}{c}\text { Proposed } \\
\text { HA(GWO-CSO) }\end{array}$ & 155 & 3.86 & $2,5,6,1,1,6,4,3,2,5,6,4,3,4,51$ \\
\hline Problem statement 2 & 176 & 28.02 & $3,1,1,4,5,3,2,5,2,1,3,1,4,5,4,4,2$ \\
\hline $\begin{array}{c}\text { Genetic } \\
\text { Algorithm }\end{array}$ & 180 & 24.23 & $1,3,5,4,2,5,3,2,5,1,4,2,4,3,3,1,2$ \\
\hline $\begin{array}{c}\text { Ant Colony } \\
\text { Optimization }\end{array}$ & 178 & 4.14 & $5,1,5,5,2,1,2,3,4,4,1,3,3,2,1,5,5$ \\
\hline $\begin{array}{c}\text { Proposed } \\
\text { HA(GWO-CSO) }\end{array}$ & \multicolumn{2}{|l}{} \\
\hline \multicolumn{2}{|c|}{}
\end{tabular}

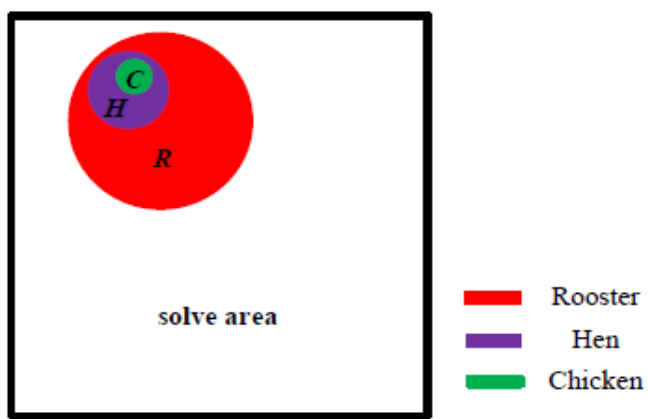

Fig. 3.:Rooster, Hen, and Chicks Food solving area

As appeared in Fig. 3, after a specific timeframe, as indicated by the current fitness of chicken, the entire chicken populace was revised to accomplish the refresh and after that again keep on searching for the ideal arrangement relying upon the conduct of the chicken.

Finally, the combination of the hybrid optimization approach selected to find out the ability to search the exact optimum. Here GWO is considered for storing each iteration and the CSO algorithm directly applied the scheduling iteration. To overcome the problems in existing standards, the recombination process is considered between the hybrid GWO and CSO. It effectively exhibits the hybrid method for the process of initialization and fitness computation. The GWO technique occupied the search optimization and detects the exact iterations.

\section{RESULT AND DISCUSSION}

The experimental test results of the proposed hybrid GWO-CSO based algorithm is simulated under the MATLAB environment. It is altered with a fitness value and its new solutions are achieved based on reducing the makespan. The compiled results with various iterations are listed in Table II.
Table- II: Hybrid Algorithm Result

\begin{tabular}{|l|l|l|l|l|l|}
\hline $\begin{array}{l}\text { Problem } \\
\text { statemen } \\
\mathbf{t}\end{array}$ & $\begin{array}{l}\text { No of } \\
\text { iterati } \\
\text { ons }\end{array}$ & $\begin{array}{l}\text { Mach } \\
\text { ines } \\
(\mathrm{m})\end{array}$ & $\begin{array}{l}\text { Jobs } \\
(\mathrm{n})\end{array}$ & Job times (j) & $\begin{array}{l}\text { Targe } \\
\mathbf{t} \\
\text { soluti } \\
\text { on }\end{array}$ \\
\hline 1 & 1000 & 6 & 16 & $\begin{array}{l}\mathbf{5 8 , 7 2 , 7 9 , 4 3 , 1 6 , 3 7 , 7 , 7 4 , 9} \\
7,44,39,80,65,65,39,96\end{array}$ & 154 \\
\hline 2 & 1000 & 5 & 17 & $\begin{array}{l}\mathbf{5 0 , 2 2 , 1 3 , 6 4 , 4 3 , 7 0 , 5 3 , 5 5 ,} \\
\mathbf{9 5 , 8 3 , 5 4 , 5 6 , 6 0 , 7 7 , 2 8 , 2 4 ,}\end{array}$ & 175 \\
\hline
\end{tabular}

The hybrid algorithms declared that makespan time is reduced. It is combined by reducing the total processing duration as allocated the iterations exactly to all traditional algorithms like GA, ACO and the proposed one.

The active job sequences offer the base makespan time for the whole process and they contain the information having time lengths which are added to those of alternate tasks. The base makespan length is accomplished toward the whole activity of the whole jobs. In case the issue is changed, and then the handling span is likewise seen to adjust as per the quantity of the machine working. These activity successions are prepared to yield the slightest makespan length. Thus, the base makespan length is accomplished in the GWO with CSO when contrasted and the GWO and CSO strategies. The difference between the proposed length and novel strategy, the term is 3.86 .

The charts appearing in figure 4 effectively demonstrate the execution examination parameters wellness diagrams in terms of the hybrid procedure with the existing GA and ACO. It is noticed that the makespan time for the issue given the cycle factors. On the off chance that the emphasis shifts, the time is diminished. In this regard, the makespan time is achieved in the HA in the 1000 cycle. When it is contrasted and the GWO, the time distinction in 1000 cycles and the base time accomplished, Hybrid GWO-CSO contrasting and others on account of the GA likewise the base time is achieved in the more emphases.

However, on account of ACO, as the quantity of emphasis level is high, it prompts the reduction in the time and finally, the arrangement identified with the lower wellness is accomplished in the particular cycle.

Hence, it is noticed that the makespan of the various algorithms is reduced. Since the proposed algorithm has the least cost when compared with the other algorithms. It is noticed that the cost of the algorithm is reduced when compared with various iterations. 


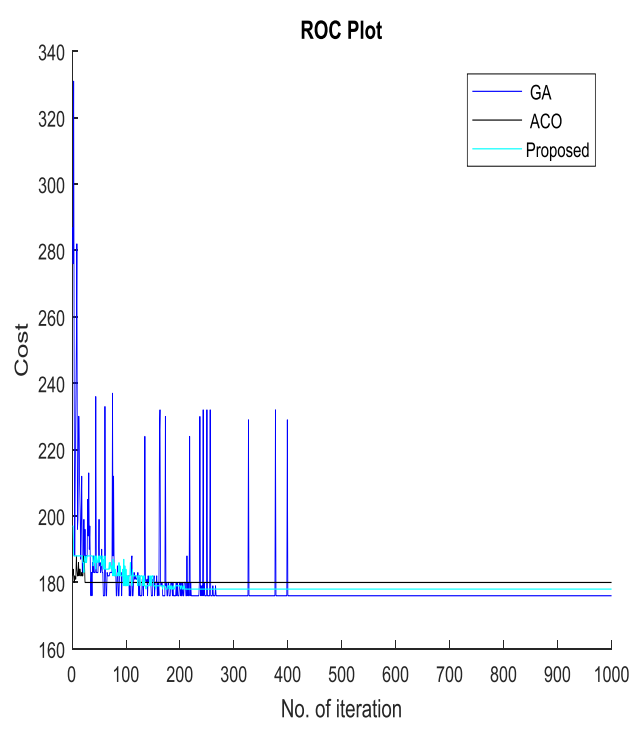

Fig. 4. Convergence graph for various algorithms

\section{CONCLUSION}

The first serious problem in industrial machinery is Job shop Scheduling. Some traditional algorithms are considered for solving such issues but still, they are not very much powerful. Since the traditional algorithms like GA are simple and efficient for scheduled job sections. Hence, this work focused on three different algorithms with various iteration to minimize makespan. The concept considered is a swarm-based algorithm aimed to observe the hybrid model with GWO as a mathematical model. The algorithm has been coded in MATLAB and executed successfully. The proposed hybrid algorithm requires very less time duration as $3.86 \mathrm{~s}$ for 1000 iterations compared with GA as 28.72 s and ACO as 26.06s. Hence, the overall cost is reduced. It is conventional and simple for complex problems as the GWO algorithm is competent to reach an exact goal. The attractive results authenticate that the pioneering Hybrid optimization algorithm proves the least makespan time.

\section{REFERENCES}

1. J. F. Gonçalves, J. J. de Magalhães Mendes, Resende, "A hybrid genetic algorithm for the job shop scheduling problem," European journal of operational research, 167, 77-95, 2005.

2. M. Kuroda, Z. Wang, "Fuzzy job shop scheduling," International Journal of Production Economics, 44, 45-51,1996.

3. F. Glover, "Heuristics for integer programming using surrogate constraints," Decision Sciences, 8(1), 156-166, 1977.

4. F. Glover, "Future paths for integer programming and links to artificial intelligence," Computers \& operations research, 13(5), 533-549, 1986.

5. W. Gu, D. Tang, K. Zheng, "Minimizing makespan in job-shop scheduling problem using an improved adaptive particle swarm optimization algorithm," In Control and Decision Conference (CCDC), pp. 3189-3193, 2012.

6. M. A. González, C. R. Vela, R. Varela, "A New Hybrid Genetic Algorithm for the Job Shop Scheduling Problem with Setup Times, " In ICAPS, pp. 116-123,2008.

7. Ponsich, C. A. Coello, "A hybrid differential evolution-tabu search algorithm for the solution of job-shop scheduling problems," Applied Soft Computing, 13(1), 462-474, 2013.

8. J. Kassu, B. Eshetie, "Job Shop Scheduling Problem for Machine Shop with Shifting Heuristic Bottleneck," Global Journal of Research In Engineering, 15, 1-7, 2015.
9. L. Cheng, Q. Zhang, F. Tao, K. Ni, Y. Cheng, "A novel search algorithm based on waterweeds reproduction principle for job shop scheduling problem," International Journal of Advanced Manufacturing Technology, 84,2016.

10. H. Zhou, Y. Feng, L. Han, "The hybrid heuristic genetic algorithm for job shop scheduling," Computers \& Industrial Engineering, 40(3), 191-200, 2001.

11. B. J. Park, H. R. Choi, H. S. Kim, “A hybrid genetic algorithm for the job shop scheduling problems," Computers \& industrial engineering, 45(4), 597-613, 2003.

12. M. Sakawa, R. Kubota, "Fuzzy programming for multiobjective job shop scheduling with fuzzy processing time and fuzzy due date through genetic algorithms, "European Journal of operational research, 120(2), 393-407, 2000.

13. T.L. Lin, S. J. Horng, T. W. Kao, Y. H. Chen, R. S. Run, R. J. Chen, I. H. Kuo, "An efficient job-shop scheduling algorithm based on particle swarm optimization," Expert Systems with Applications, 37(3), 2629-2636, 2010.

14. R. Zhang, S. Song, C. Wu, "A hybrid artificial bee colony algorithm for the job shop scheduling problem," International Journal of Production Economics, 141(1), 167-178, 2013.

15. D. Y. Sha, H. H. Lin, "A multi-objective PSO for job-shop scheduling problems," Expert Systems with Applications, 37(2), 1065-1070, 2010.

16. Muthiah, A. Rajkumar, R. Rajkumar, "Hybridization of artificial bee colony algorithm with particle swarm optimization algorithm for flexible job shop scheduling," International Conference on Energy-Efficient Technologies for Sustainability (ICEETS), 896-903, 2016

17. W. Wisittipanich, V. Kachitvichyanukul, "Two enhanced differential evolution algorithms for job shop scheduling problems," International Journal of Production Research, 50(10), 2757-2773, 2012.

18. M. Ziaee, "Job shop scheduling with makespan objective: A heuristic approach," International Journal of Industrial Engineering Computations, 5(2), 273-280, 2104.

19. P. C. Ma, F. Tao, Y. L. Liu, L. Zhang, H.X. Lu, Z. Ding, "A hybrid particle swarm optimization and simulated annealing algorithm for job-shop scheduling" IEEE International Conference on Automation Science and Engineering (CASE), 125-130, 2014.

20. L. Asadzadeh, "A local search genetic algorithm for the job shop scheduling problem with intelligent agents," Computers \& Industrial Engineering, 85, 376-383, 2015.

21. B. Liu, Y. Fan, Y. Liu, "A fast estimation of distribution algorithm for dynamic fuzzy flexible job-shop Scheduling problem," Computers \& Industrial Engineering, 87, 193-201, 2015.

22. H. C. Chang, Y. P. Chen, T. K. Liu, J. H. Chou, "Solving the flexible job shop scheduling problem with makespan optimization by using a hybrid Taguchi-genetic algorithm," IEEE Access, 3, 1740-1754, 2015.

23. X. Meng, Y. Liu, X. Gao, H. Zhang, "A new bio-inspired algorithm: chicken swarm optimization," In International conference on swarm intelligence, 86-94, 2014.

24. M. K. Marichelvam, P. Manimaran, M. Geetha. "A Hybrid Genetic Scatter Search Algorithm to solve Flexible Job Shop Scheduling Problems," Soft Computing Techniques and Applications in Mechanical Engineering, 181-194, 2018. 


\section{AUTHORS PROFILE}

S. Kavitha , received her Bachelor degree in Mechanical Engineering from Bharathiyar University, Coimbatore, India and Masters in CAD from Sathyabama University, Chennai, India. She is currently pursuing her $\mathrm{PhD}$ in Kalasalingam University, Tamilnadu, India. She is also orking as an Assistant Professor in the Department of Mechanical Engineering, Kalasalingam University. Her research interests include operations management and scheduling.

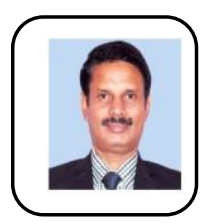

Dr. P. Venkumar, is currently a Senior Professor in Department of Mechanical Engineering, Kalasalingam Academy of Research and Education, Krishnankovil, Tamilnadu, Inida. He received his B.E., and M.E., degrees in Thigagrajar College of Engineering, Madurai, Tamilnadu, India, in 1994 and 1997, respectively and Ph.D. degree in Manonmaniam Sundaranar University, India in Industrial Engineering in 2006. His main areas of research interest are Manufacturing system optimization, Supply chain management and Smart manufacturing. 